Pensamiento Crítico N. ${ }^{\circ}$ 9, pp. $11-26$

\title{
La percepción del riesgo y su relación con la actual crisis financiera global
}

Mg. Jorge Barrera Herrera

\section{RESUMEN}

El objetivo de este artículo es analizar los principales factores de la excesiva prociclicidad de los sistemas financieros, debido al incremento innecesario de las oscilaciones en el nivel de actividad lo cual pone en riesgo la estabilidad financiera con repercusiones negativas en la economía real de los países.

El normal crecimiento económico real de un país va en paralelo con el crecimiento de los sistemas financieros en general y con el crecimiento de los créditos en particular, ocasionando una prociclicidad lógica y deseable muy fuerte entre estos, siempre poniendo en evidencia como las balanzas de pagos nacionales se corresponden como elementos interdependientes de un sistema monetario global de suma cero.

La problemática se plantea en la medida en que existen factores inherentes al comportamiento de los agentes económicos que actúan dentro de los sistemas financieros o que se derivan de la regulación prudencial que aumentan la volatilidad en forma anormal respecto de sus niveles considerados como fundamentales, teniendo una repercusión procíclica de amplitud en el ciclo económico, siempre inconveniente por la generación de inestabilidad y turbulencia en los precios. 


\section{Jorge Barrera Herrera}

La actual crisis financiera alcanza magnitudes de pérdidas sin precedentes, y todavía está cobrando víctimas, es claro que la función de riesgos de las entidades financieras jugará un papel clave, adaptando su gestión a los mercados, considerando los aspectos conductuales y de percepción de los agentes económicos. Con relación a la percepción del riesgo y al crédito, un aspecto preocupante en el escenario actual es cómo finalmente serán afectados los dos canales principales de financiación del sistema financiero mundial, el canal del crédito bancario y la financiación en los mercados de capitales.

Es necesario, hoy en día, probar que los beneficios esperados, sobre los que se sustenta la valoración financiera, realmente pueden efectivizarse, dado que se basan en la ganancia que se extraería del futuro como producto de la especulación más que de el valor generado por el trabajo realmente productivo.

Palabras clave: Finanzas conductistas, titulización, regulación prudente, volatilidad, prociclicidad, hipótesis de eficiencia de los mercados financieros, comportamiento de rebaño, función de riesgos, crisis financiera, burbuja financiera.

\section{ABSTRACT}

The aim of this article is to analyse the main factors of excessive prociclicidad of financial systems, due to increased unnecessary fluctuations in the level of activity which puts at risk the financial stability with a negative impact on the real economy of the countries.

The normal real economic growth of a country goes in parallel with the growth of financial systems in general and with the growth of appropriations in particular, causing a logical and desirable prociclicidad very strong among these, always putting in evidence as the balances of payments National correspond as interdependent elements of a global monetary system zero-sum. The problem arises to the extent that there are factors inherent in the behaviour of economic agents acting within the financial systems or arising out of prudential regulation which increase volatility in abnormal form on their levels considered fundamental to have a procyclical impact amplitude in the economic cycle, always inconvenient for the generation of turbulence and volatility in prices.

Keywords: Presential regulation, financial behaviour, volatility. 


\section{La percepción del riesgo y su relación con la actual crisis financiera global}

\section{INTRODUCCIÓN}

Hay suficiente evidencia de que cuando las condiciones económicas son favorables y la valoración de los activos (financieros o reales) es alta, se produce un crecimiento fuerte de los créditos, una mayor predisposición a asumir riesgos y un impulso de la actividad económica, la prociclicidad en el ámbito financiero; por tanto, es un resultado natural e incluso deseable.

Sin embargo, la excesiva volatilidad financiera, tanto en la fase alcista del ciclo económico como en la bajista, la cual difiere de la volatilidad fundamental, genera, necesariamente, riesgos de corrección muchas veces desordenados e intempestivos, tales como los excesos de sobreendeudamiento y restricciones de los créditos del sistema bancario y del mercado de capitales (disponibilidad de liquidez), comprometiendo la estabilidad financiera y también -lo que es más importante aún- al crecimiento de la economía real del país, dada la gran correlación positiva entre estas.

Estos hechos nos plantean algunas interrogantes que ponen en discusión las hipótesis de eficiencia de los mercados financieros y el comportamiento de los agentes racionales $^{1}$, dando relevancia a las finanzas conductistas ${ }^{2}$.

Este trabajo, en términos generales, se ha desarrollado dando énfasis a las finanzas conductistas que estarían explicando algunos patrones de comportamientos constatados en la reciente crisis financiera; en el segundo epígrafe, se describe y explica el comportamiento de los agentes económicos en cuanto a la prociclicidad entre crecimiento económico, el crédito, la volatilidad financiera y la regulación; en el tercer epígrafe, se describe la actual crisis financiera, sus causas y el efecto procíclico; en el cuarto epígrafe, se analizan las principales causas de la prociclicidad del crédito; $y$, finalmente, en el último apartado, se muestran las principales conclusiones.

\section{PROCICLICIDAD, EL CRÉDITO, VOLATILIDAD FINANCIERA Y REGU- LACIÓN PRUDENCIAL}

Los cambios en la percepción y actitud frente al riesgo de los agentes económicos, están íntimamente ligados al estado de la economía y, por ende, a los ciclos económicos.

1 Mercado eficiente es aquel en el cual los precios siempre reflejan plenamente toda la información disponible.

2 Estudio de cómo los inversionistas cometen errores de juicio sistemáticamente. 


\section{Jorge Barrera Herrera}

En ciclos expansivos de bonanza económica el grado de optimismo suele incrementarse, reduciéndose la percepción del riesgo e incrementándose en consecuencia la exposición al mismo, produciéndose un efecto inverso más potente en el caso cuando el ciclo económico cambia contractivamente agravando el pesimismo de los agentes.

Este comportamiento de los agentes económicos, que se aparta de lo establecido en las hipótesis de eficiencia de los mercados financieros y de la conducta racional, se originaría en la asimetría en la información entre prestatarios y prestamistas, y en la interrelación entre la evolución del crédito y la de los precios de los activos que actúan como colaterales.

En una fase expansiva del ciclo económico, se fomentan condiciones favorables para invertir y los procesos de valoración de activos (financieros o reales) presionan al alza $^{3}$, suele aumentar el optimismo de los agentes; y como consecuencia, se produce un fuerte crecimiento del crédito del sistema bancario y mercado de capitales (algunos no regulados o informales), con una mayor predisposición a asumir riesgos lo cual alimenta e impulsa la actividad económica.

Como consecuencia y a medida que los precios de los activos crecen, el cociente de deuda sobre riqueza tiende a disminuir, lo que aumenta la capacidad de los agentes para ser sujetos de crédito y si el nuevo endeudamiento se dirige a la compra de activos, el valor de estos tiende a aumentar aún más.

De esta manera, la relativa estabilidad del ratio deuda sobre riqueza encubre un inminente deterioro en la relación deuda sobre ingresos, lo cual -en definitiva- hace descansar la sostenibilidad del endeudamiento en el largo plazo, sobre la elevada valoración de los activos que en buena cuenta actúan como colaterales del préstamo.

A medida que se produjeran aumentos de los precios, estos podrían validar o incluso generar unas expectativas de beneficios irrealistas, ${ }^{4}$ acrecentándose el carácter especulativo del mercado financiero.

3 Los precios de los activos ejercen una influencia significativa sobre la evolución del crédito, y viceversa.

4 En la medida que las valoraciones no se sustenten en sus flujos netos de rendimientos esperados. Véase, Borio, Kennedy y Prowse (1994). 


\section{La percepción del riesgo y su relación con la actual crisis financiera global}

Después, cuando el ciclo económico cambia y se vuelve contractivo y se hace evidente que no se podrán satisfacer las expectativas de rentabilidad compatibles con los altos precios alcanzados por los activos (burbujas financieras), se produce una contracción del crédito en forma indiscriminada, incluso para proyectos de inversión rentables y, simultáneamente, también se produce una abrupta y, a veces, desordenada corrección de las sobrevaloraciones de los instrumentos financieros en los mercados de capitales.

La consecuencia final de todo este proceso, es la generación de una mayor volatilidad financiera, tanto en la fase alcista del ciclo económico como en la bajista, creándose la posibilidad, en las recesiones económicas, de la aparición de crisis financieras que fomenten a su vez, desaceleraciones económicas reales, dada la alta correlación positiva entre estas.

Una tentación, cuando los problemas financieros se transforman con celeridad en dificultades para la economía real, cuyos efectos negativos sobre el bienestar ciudadano son evidentes, es tratar de resolver dichos problemas mediante un incremento de la regulación.

La regulación debe entenderse como un proceso a través del cual es posible identificar las modificaciones necesarias a introducir en el sistema, en respuesta a una realidad económica cambiante. No obstante, plantear nuevas normas aumenta el riesgo de que el resultado final implique una sobrerregulación que afecte el libre desenvolvimiento de los mercados, generando ineficiencias, afectando la competitividad; la tarea es, pues, conseguir el equilibrio necesario.

\section{A PROPÓSITO DE LA ACTUAL CRISIS FINANCIERA GLOBAL}

Los históricos déficit, fiscal y comercial, creciente de la economía norteamericana, han permitido que este actúe como motor del consumo que alimentó el auge de la economía mundial, actualmente su estrategia competitiva se basa en una progresiva devaluación del dólar.

Existe un gran crecimiento del capital destinado a las finanzas, este crecimiento de la liquidez se ha triplicado entre el 2000 a 2007, en comparación con el capital dedicado a la producción de bienes y prestación de servicios de la economía real. 


\section{Jorge Barrera Herrera}

La creación de valores respaldados por hipotecas y otros préstamos e innovaciones hicieron más fácil que las compañías financieras distribuyeran el riesgo y estuvieran más dispuestas a otorgar préstamos a familias para incentivar el gasto. Las deudas de las familias en Norteamérica, incluyendo hipotecas y tarjetas de crédito, pasaron del 13\% del total de los activos en 1980 al 19\% en el 2007. En ese período, la tasa de ahorro personal cayó a casi cero.

La actual crisis financiera global comenzó en un segmento definido del mercado hipotecario estadounidense, el cual es poco regulado (créditos subprime); logrando contagiar, con una velocidad sorprendente al sistema financiero internacional (mercado interbancario internacional), forzando a distintos bancos centrales a intervenir de forma masiva, concertada y creativa.

Edgard M. Gramlich (2007), estudioso del mercado de hipotecas subprime, establece que la crisis en este segmento de negocio es comparable a los procesos de expansión y corrección que habitualmente van asociados a los procesos de incremento de la productividad, los cuales no tienen un desarrollo lineal, sino que tienden a combinar fases de rápida expansión y euforia con importantes correcciones posteriores.

Los antecedentes de esta crisis se remontan a los años 2001, cuando la Reserva Federal Americana (FED), para contrarrestar los efectos negativos sobre la economía americana de la caída del mercado de acciones arrastrado por el colapso de las expectativas creadas alrededor de las empresas de servicios por Internet y tecnologías afines, establece los tipos de interés de referencia para su economía en mínimos históricos, en promedio al $2 \%$ durante casi tres años, propiciando un período de revalorización continua de activos inmobiliarios. Desde el 2001 al 2004, inclusive, fue un período de gran moderación y estabilidad económica, como de exceso de ahorro para la mayoría de economías en el mundo en general, en donde se destacan los bajos niveles de tasas de interés de referencias y reducida volatilidad, lo que contribuyó al crecimiento del apalancamiento en el sistema financiero, a la minusvaloración de los riesgos y a la sobrevaloración de algunos activos.

A partir del 2004, la FED decidió aumentar, gradualmente, los tipos de interés de referencia, afectando al ritmo de crecimiento de los precios de la vivienda. Cuando a 


\section{La percepción del riesgo y su relación con la actual crisis financiera global}

mediados del 2007, los tipos de interés de referencia alcanzan niveles del $5 \%$, las variables macroeconómicas fuerzan a la baja la revisión de las expectativas para la fijación de precios del sector inmobiliario, incrementándose, paralelamente, los niveles de mora de pagos de las deudas. Esto provoca el endurecimiento de las condiciones exigidas, al otorgar nuevos créditos y el aumento de los spreads comerciales, desencadenando un espiral de falta de pagos en este sector. Los fondos de cobertura y los bancos comenzaron a tener dificultades de liquidez, las primas de los "swaps" de créditos empezaron a dispararse y las agencias calificadoras de créditos comenzaron a rebajar las calificaciones de activos que previamente eran considerados como de la mejor calidad.

Otro factor estructural de la presente crisis financiera, además de la falta de liquidez, está ligado a la creciente innovación financiera y su reflejo en el aumento del volumen de operaciones en derivados de crédito, lo que facilita a las entidades financieras a transferir o adquirir, segmentos diferenciados del riesgo de crédito en sus balances. Una buena parte de los créditos subprime no estaban concentrados en bancos con balances razonablemente diversificados, sino segmentados y repartidos en estructuras empaquetadas y revendidas a otros agentes del mercado financiero, tales como hedge funds, aseguradoras, inversores privados $\mathrm{u}$ otros bancos.

En resumen, el crecimiento del apalancamiento, a partir del 2001 hasta la evidencia de la crisis a mediados del 2007, se ha producido a través de una acumulación de riesgos en los sistemas financieros mucho más rápida que el crecimiento de la base de capital que debe cubrir las pérdidas cuando estas se materializan. Este apalancamiento ha sido explicito en lo relativo a los préstamos del sistema bancario y a la emisión de deuda.

Sin embargo, la provisión de capital que debe cubrir las pérdidas de los nuevos instrumentos financieros, derivados, titulización, productos estructurados, vehículos de inversión estructurados (SIV, estructuras fuera de balance) se efectuó en forma poco rigurosa para la eventualidad de proteger posibles contingencias.

La actual crisis financiera alcanza magnitudes de pérdidas sin precedentes y todavía está cobrando víctimas, es claro que la función de riesgos de las entidades financieras jugará un papel clave, adaptando su gestión a los mercados, aportando una visión integrada frente a las visiones segmentadas en que cada riesgo es gestionado aparte de los demás. Se deberá regular e implantar políticas prudentes de 


\section{Jorge Barrera Herrera}

provisiones y de asignación de capital para asegurar un margen de maniobra adecuado y cubrir eventualidades inesperadas. En este sentido, el capital se destaca como la herramienta de integración de riesgos, y la gestión avanzada del mismo se basa en la optimización de este recurso y en la obtención de los retornos adecuados a los niveles de riesgo asumidos, lo que garantizaría la creación de valor en los negocios.

\subsection{Detalles sobre el origen de las tensiones de liquidez}

En julio de 2006 se producen los primeros momentos de tensión en norteamérica, cuando el índice de morosidad de los préstamos hipotecarios sufre un cambio de tendencia al alza, de los denominados préstamos subprime $e^{5}$ y se agudizan cuando se conoce que muchos de ellos han sido titulizados, de forma que estos activos han sido adquiridos por vehículos de inversión (SIV) que se financiaban en el mercado mediante emisiones de títulos a corto plazo (conocidos como asset backed commercial paper o $\mathrm{ABCP}$ ). Estos vehículos, habitualmente, eran creados y patrocinados por entidades de crédito que, a la vez, solían otorgarles líneas de liquidez con el fin de mejorar la calidad crediticia de las emisiones. Un banco puede establecer un vehículo de inversión (SIV) siempre y cuando sus socios estén dispuestos a tener beneficios y pérdidas máximas por los cambios proyectados en el valor de los instrumentos subyacentes. A los bancos no se les pide revelar los activos que venden a sus propias SIV, ni el precio pagado, ni si su valor ha disminuido, lo que dificulta a los inversores determinar cuándo terminará la crisis "subprime".

En agosto de 2007, se confirma la tendencia a la baja del valor de los inmuebles, las dudas sobre la valoración de los activos que formaban las tenencias de estos vehículos llevaron a los inversores a corto plazo a retirarse del mercado de ABCP, al no poder refinanciar, obligó a los vehículos (SIV) a usar las líneas de liquidez. Por lo anteriormente expuesto, se generó el temor a que los bancos tengan que sacar activos de las SIV y volverlos a colocar en sus estados financieros, lo que significaría el reconocimiento de nuevas pérdidas.

La actuación de los inversores en este período de turbulencia financiera, ha servido de amplificador de los problemas de prociclicidad y volatilidad, pues al materializarse

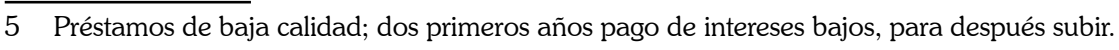




\section{La percepción del riesgo y su relación con la actual crisis financiera global}

las dudas sobre el valor de algunos activos y sobre la solvencia de algunas entidades, su movimiento natural hacia los valores alejados de las dificultades (fundamentalmente deuda soberana, deuda de empresas de primera calidad, commodities) ha afectado, aún más, los valores de los activos que se desprendían, produciéndose un espiral de consecuencias negativas, al generarse caídas significativas en su valoración acentuando la posición de retirada.

\section{FACTORES DE LA PROCICLICIDAD FINANCIERA}

Dos son las principales causas que podrían subyacer a la excesiva prociclicidad del crédito:

- El protagonismo y nuevas características de la medición del valor, y, especialmente, del riesgo en las decisiones propias de la actividad bancaria.

- Reacciones de los agentes a las variaciones en la percepción del riesgo.

\subsection{El protagonismo y nuevas características de la medición del valor y espe- cialmente del riesgo en las decisiones propias de la actividad bancaria}

El desarrollo de modelos para la medición y gestión del valor y riesgo ha supuesto uno de los avances más notables en el sistema financiero internacional durante los últimos años.

El sistema bancario, principalmente, ha reforzado sus procesos de toma de decisiones con la aplicación de modelos, con el fin de valorar mejor los nuevos instrumentos financieros, para la concesión de créditos, la determinación del nivel de provisiones o la asignación de capital, esto ha supuesto un incremento en la eficiencia de la actividad financiera teniendo un impacto positivo en la economía real.

Sin embargo, en el marco de la prociclicidad financiera, estos beneficios no están exentos de costes potenciales si los modelos infravaloran el riesgo en las épocas de bonanza y lo sobreestiman en las recesiones, debido a que la medición y evolución del riesgo depende del horizonte temporal utilizado para el computo de algunos parámetros, como de la atención prestada a los cambios en las correlaciones entre las variables en el tiempo. 


\section{Jorge Barrera Herrera}

\subsubsection{Gestión de riesgos}

La innovación financiera ha permitido llevar a cabo una gestión del riesgo de crédito más eficiente, al desplazar el riesgo desde sus originadores hasta terceros más dispuestos a asumir mayores riesgos, y porque disminuyen los costes de endeudamiento.

Las fallas en las instituciones financieras han sido debidas tanto a deficiencias en los modelos cuantitativos que facilitaban la toma de decisiones como a las debilidades detectadas en las estructuras de gobierno y en los procesos de toma de decisiones y de control de riesgo.

Es posible que tanto la regulación prudencial como las normas contables proporcionen un conjunto de incentivos que ayuden a una adecuada gestión avanzada del riesgo y de refuerzo en la coordinación internacional. El aspecto más relevante de Basilea II, quizá no resida en el Pilar 1, que establece las exigencias mínimas de capital regulatorio, sino en el Pilar 2 , que supervisa los métodos y modelos internos empleados por las entidades para la determinación del "capital económico", capital necesario según el perfil del riesgo, estableciendo objetivos estratégicos de capital en función de los planes futuros de negocio.

\subsubsection{Valoración, normas contables y transparencia}

La valoración a precios de mercado de productos complejos y no transparentes, en mercados poco líquidos, y cuyo activo subyacente (la vivienda) ha sufrido un continuo deterioro de valor, ha generado una dinámica descendente de los precios y de valoraciones muy corrosiva para los balances de las instituciones financieras.

Los agentes económicos podrán mejorar sus procesos de valoración de la calidad crediticia de las instituciones con las que negocian, si estas revelan sus interrelaciones con los vehículos de propósito especial (SIV) fuera de balance.

Las exigencias de información deben avanzar al mismo ritmo con el que se desarrollan los mercados financieros, esto se está mejorando tanto por los nuevos estándares contables IFRS, así como por el Nuevo Acuerdo de Capitales Basilea II. 


\section{La percepción del riesgo y su relación con la actual crisis financiera global}

\subsubsection{Modelos de banca y la innovación financiera}

La visión clásica del riesgo era la sensibilidad del valor de los instrumentos respecto a unas variables explicativas, que suelen ser confiables, accesibles y bien medidas (tipos de interés, tipos de cambio), el riesgo principal es el riesgo de contrapartida. En cambio, la actual visión del riesgo es que se puede crear un mercado donde, a través de productos sintéticos basados en la diversificación, haya inversores que compren carteras hipotecarias con una calidad que el prestamista original no está dispuesto a aceptar. Aquí las variables explicativas son más sutiles, como la probabilidad de impago de una contrapartida, la correlación, la subordinación de un tramo de una cartera.

Desde el modelo tradicional de gestión bancaria hold-to- maturity, en el que se otorgaban préstamos que permanecían en el balance hasta vencimiento (con repago o cancelación), se ha pasado a una estrategia más orientada hacia originate-to distribute, es decir, estructurar la financiación otorgada de tal forma que no permanece en los libros, sino que se desplaza fuera del balance.

Cuando el préstamo se otorga con la idea de no mantenerlo en el balance, lo que prima es la expectativa de venderlo en el mercado, por lo que existen menos incentivos para utilizar los mismos criterios de seguridad y prudencia que si los mantuviesen dentro de sus balances hasta el vencimiento. A pesar de que el modelo originate-to-distribute es más flexible y eficiente; lo anteriormente expuesto es una de sus evidentes debilidades.

\subsubsection{Gestión de liquidez por parte de las instituciones y bancos centrales}

El riesgo de liquidez del mercado se refiere a la dificultad de una institución financiera para deshacer posiciones sin que se vea afectado de forma significativa el precio de mercado, bien porque el mercado no es suficientemente profundo, o bien por una interrupción en el mismo.

En un contexto globalizado con crecientes operaciones cross-border, instrumentos innovadores complejos fuera de balance que incluyen opcionalidad y mayor interdependencia entre mercados, hay que mantener la capacidad de intercambio de valores tanto en condiciones normales como de crisis. Cuando aumenta el riesgo de crédito y de mercado, los mercados se vuelven más volátiles, ocasionando una limitación de activos 


\section{Jorge Barrera Herrera}

y un despalancamiento, lo que amplia los spreads de crédito de aquellos activos con más riesgo.

La ausencia de precios y de mercados secundarios para algunos productos de crédito, así como la localización y el tamaño de las pérdidas potenciales, conducen a restricciones en algunos mercados monetarios y a dificultades de financiamiento para un gran número de instituciones financieras, puesto que algunas se muestran renuentes a dar crédito a los que, en su opinión, tienen baja calidad o bien activos ilíquidos; justamente estos productos crediticios fueron usados como colaterales en sus endeudamientos por parte de los bancos y hedge funds en la actual crisis financiera.

La desaceleración económica global con grandes pérdidas bancarias reportadas y una explosión de fondos que pueden beneficiarse de las caídas de las cotizaciones en los mercados secundarios, está alentando el aumento de ventas en corto, contribuyendo a enviar a 22 de los 23 países incluidos en el MSCI World Index a mercados bajistas, práctica que implica tomar prestado valores para venderlos con la expectativa de que pueden ser recomprados a un precio más bajo antes de devolver el crédito anterior.

Para aliviar la situación de iliquidez, en los mercados interbancarios, los bancos centrales tuvieron que inyectar liquidez de forma extraordinaria y, así, facilitar el funcionamiento ordenado de los mercados, es necesario mencionar también la participación muy importante de los fondos soberanos.

\subsection{Reacciones de los agentes a las variaciones en la percepción del riesgo}

Cuando los inversores siguen en forma masiva (efecto manada o comportamiento de rebaño) estrategias parecidas para deshacerse de las posiciones en productos similares, los precios se desploman y algunos fondos se declaran en quiebra generándose una escasez de efectivo, esto es fomentado por la adopción simultánea de modelos de medición y gestión del riesgo "similares" por parte de los agentes, lo cual incrementa la prociclicidad financiera, esto se podría dar incluso si no existiera una sobre reacción individual o aunque la medición del riesgo fuera correcta. Existen, además, factores propios del sistema financiero que incentivan respuestas masivas, tales como: 


\section{La percepción del riesgo y su relación con la actual crisis financiera global}

- En la medida en que todos hagan lo mismo, se configura un proceso de expectativa autoconfirmada, en virtud del cual ratifica la decisión aumentando la volatilidad del ciclo financiero.

- Los sistemas de retribución basados en la consecución de objetivos a corto plazo, no necesariamente sostenibles en el tiempo, también favorecen exposiciones al riesgo claramente procíclicas.

- La creciente competencia también podría inducir a las instituciones a incrementar su nivel de riesgo, aun cuando su medición del riesgo fuera correcta y su aversión al mismo no menguara, en la medida en que se vieran obligadas a mantener un determinado tamaño en el negocio.

- Los sistemas de retribución de los gestores basados en una medición de los resultados relativa respecto a los obtenidos en el conjunto del sistema financiero (no en términos absolutos) incrementan el comportamiento de rebaño de los participantes, ya que penalizan los resultados negativos solo en la medida en que diverjan de la media de resultados del sector. Así, se generan incentivos a gestionar de forma pasiva la cartera de activos, siguiendo índices de referencia, sin incorporar el análisis de los fundamentos.

- Los delitos siguen a la economía y al desarrollo de los mercados financieros, en épocas expansivas la conducta de los agentes se vuelve proclive al uso de información privilegiada, en tanto la economía se desacelera la tendencia se centra en los fraudes contables y relacionados.

- Los agentes económicos tienden a sobre estimar lo bien que se sentirían ante noticias buenas, ciclos expansivos económicos y lo devastador que nos dejaría una noticia mala o de una recesión, lo que se denomina el prejuicio del impacto, sobre estimando o subestimando la intensidad y duración de las emociones, lo que puede llevar a cometer errores en la toma de decisiones de inversión.

- Para la toma de decisiones es básico los esfuerzos de predicción, al cerebro humano le encantan los patrones, los vemos incluso donde no existen, logrando que tengan algún sentido, la respuesta es subconsciente y automática; lo cual incita, a veces, a decisiones de carácter poco realistas. 


\section{Jorge Barrera Herrera}

- Cuando el crecimiento económico de un país aumenta, la satisfacción de la población se reduce, al menos, inicialmente, esta insatisfacción (que es una paradoja) parece resultar de las crecientes expectativas y aspiraciones personales cuando aumenta el ingreso de los demás, produciéndose una disociación entre la realidad objetiva del crecimiento y la percepción subjetiva de la satisfacción ${ }^{6}$.

\section{CONCLUSIONES}

El normal crecimiento económico real de un país va en paralelo con el crecimiento de los sistemas financieros en general y con el crecimiento de los créditos en particular, ocasionando una prociclicidad lógica y deseable muy fuerte entre estos, siempre poniendo en evidencia como las balanzas de pagos nacionales se corresponden como elementos interdependientes de un sistema monetario global de suma cero.

La problemática se plantea en la medida en que existan factores inherentes al comportamiento de los agentes económicos que actúan dentro de los sistemas financieros o que se derivan de la regulación prudencial que aumentan la volatilidad en forma anormal respecto de sus niveles considerados como fundamentales, teniendo una repercusión procíclica de amplitud en el ciclo económico, siempre inconveniente por la generación de inestabilidad y turbulencia en los precios.

El auge en las innovaciones financieras, en especial de la titulización de activos, está íntimamente ligada con las ventajas que aporta a los inversionistas, a los que ofrece combinaciones de rentabilidad-riesgo antes no existentes, ya que posibilita nuevas vías de captación de financiamiento, así como de gestión de los riesgos, se espera un desarrollo menor y su gestión sufrirá algunas mejoras. De la misma manera, los derivados estructurados seguirán ofreciendo nuevas y más eficientes formas de gestión, pero existirá un mejor conocimiento y uso de los mismos.

La actual crisis financiera alcanza magnitudes de pérdidas sin precedentes y todavía está cobrando víctimas, actuales discrepancias en las tasas de corto plazo revelan que todavía hay preocupación sobre la salud de los bancos; un indicador, es el hecho de que la brecha entre las tasas de interés de referencia de los bancos centrales y la tasa

6 Estudio del Departamento de Investigaciones del BID, basado en la encuesta mundial de Gallup sobre calidad de vida. 


\section{La percepción del riesgo y su relación con la actual crisis financiera global}

interbancaria de Londres (reflejo de la tasa que los bancos se prestan entre sí) esté aumentando y se ubique en niveles por encima del 0.10 puntos porcentuales de la brecha antes de la crisis; esto socava los esfuerzos de los bancos centrales para estimular la economía mediante la reducción de las tasas de interés.

Es claro que la función de riesgos de las entidades financieras jugará un papel clave, adaptando su gestión a los mercados, considerando los aspectos conductuales y de percepción de los agentes económicos, aportando una visión integrada frente a las visiones segmentadas en que cada riesgo es gestionado aparte de los demás.

Se deberá regular e implantar políticas prudentes de provisiones y de asignación de capital para asegurar un margen de maniobra adecuado y cubrir eventualidades inesperadas. En este sentido, el capital se destaca como la herramienta de integración de riesgos, y la gestión avanzada del mismo se basa en la optimización de este recurso y en la obtención de los retornos adecuados a los niveles de riesgo asumidos, lo que garantizaría la creación de valor en los negocios.

Con relación al crédito, un aspecto preocupante en el escenario actual es como finalmente serán afectados los dos canales principales de financiación del sistema financiero mundial, el canal del crédito bancario y la financiación en los mercados de capitales, del resultado final dependerá su efecto sobre la economía real global, situación por la cual el mundo está ya advertido. En las actuales perturbaciones, y dada la inoperancia de las instituciones internacionales tales como: FMI, BM y de los fondos de cobertura, los bancos centrales deberán hacer sus mejores esfuerzos de diagnóstico y aplicación de medidas monetarias que sin afectar o mejor promuevan el crecimiento económico, se cumplan con las metas de inflación establecidas además también se deberá considerar la importante intervención positiva que están teniendo los fondos soberanos.

Es necesario, hoy en día, probar que los beneficios esperados, sobre los que se sustenta la valoración financiera, realmente pueden efectivizarse, dado que se basan en la ganancia que se extraería del futuro como producto de la especulación más que de el valor generado por el trabajo realmente productivo.

Hoy, los mercados de commodities, están empezando a ser más difíciles de leer pues no solo las consideraciones de oferta y demanda real están detrás de los precios, 


\section{Jorge Barrera Herrera}

sino que también están siendo utilizados como instrumentos financieros, y, por lo tanto, sus precios ahora empiezan a depender de factores más especulativos, los cuales pueden crear burbujas que se inflan para después desvanecerse abruptamente.

La historia ha demostrado que no hay mejor alternativa para medir la escasez y generar precios que los mercados, pero estos harán mejor su trabajo si se logra encontrar un equilibrio apropiado y necesario para no sobrerregular y contribuir a un sistema financiero internacional más coordinado y eficiente.

\section{BIBLIOGRAFÍA}

Alonso Javier (05-2008). "Los déficits estructurales de liquidez y las tensiones en los mercados monetarios". Estabilidad Financiera N. ${ }^{\circ}$ 14, Banco de España.

Bernanke, B (2007). "Financial regulation and the invisible hand", discurso New York University Law School

Borio C.Furfine y Lowe P. (2001). "Procyclicality of the financial system and financial stability", BIS papers.

Catarineu Eva y Pérez Daniel (05-2008). "La titulización de activos por parte de las entidades de crédito". Estabilidad Financiera N. ${ }^{\circ}$ 14, Banco de España.

Del Río Pedro (05-2008). "Comparación histórica de episodios de turbulencias financieras globales". Estabilidad Financiera N. ${ }^{\circ}$ 14, Banco de España.

Sánchez Ferrero Francisco y Yanes Luciani Juan (05-2008). "De los modelos de banca y la función de riesgos”. Estabilidad Financiera N. ${ }^{\circ}$ 14, Banco de España. 